\title{
Laparoscopic liver resections at the gates of 2020: a stand-alone field of hepatobiliary surgery
}

\author{
Federica Cipriani, Francesca Ratti, Luca Aldrighetti \\ Hepatobiliary Surgery Division, IRCCS San Raffaele Hospital, Via Olgettina 60, 20132 Milan, Italy \\ Correspondence to: Federica Cipriani, MD. Hepatobiliary Surgery Division, IRCCS San Raffaele Hospital, Via Olgettina 60, 20132 Milan, Italy. \\ Email: cipriani.federica@hsr.it. \\ Provenance and Peer Review: This article was commissioned by the editorial office, Hepatobiliary Surgery and Nutrition. The article did not undergo \\ external peer review. \\ Comment on: Cai X. Laparoscopic liver resection: the current status and the future. Hepatobiliary Surg Nutr 2018;7:98-104.
}

Submitted Oct 25, 2019. Accepted for publication Nov 23, 2019.

doi: 10.21037/hbsn.2019.11.07

View this article at: http://dx.doi.org/10.21037/hbsn.2019.11.07

We read with interest the review entitled "Laparoscopic liver resection: the current status and the future" written by Dr. Xiujun Cai and published in Hepatobiliary Surgery and Nutrition (1). The author has to be commended for the excellent and updated excursus on the actual role and future perspectives of laparoscopic liver resections (LLR). The manuscript summarizes well the most acknowledged indications, procedures, techniques and concepts related to the learning process, and highlights the still ongoing issues and open debates on the topic. In this article, we aim to add few considerations and highlight some latest evidences to facilitate an even more comprehensive knowledge of the readers.

The author has started by pointing out the relevance of the first and second consensus on LLR, respectively held in Louisville (USA) in 2008 and in Morioka (Japan) in 2014. These have been essential in opening the road to the feasibility of LLR for selected indications and procedures, and stressing the importance of reaching adequate level of evidence on important elements as their efficacy, longterm outcomes, cost-effectiveness and learning curves. It is worth mentioning a further milestone that took place in Southampton (UK) in 2017, when the first international guidelines on LLR were released by a pool of eminent experts after a Delphi reiterative process to ensure a collective agreement (2). The accumulated evidence on indications, complex patients, procedures, techniques and implementation allowed to draw recommendations to prompt a safe and efficient uptake of LLR worldwide. Moreover, in 2018 two additional international consensus (on robotic liver surgery and laparoscopic living donor hepatectomy) produced specific statements on these procedures $(3,4)$. These successful and dedicated events are the demonstration that LLR have become a stand-alone growing field that deserves both a specific insight as well as full integration in the traditional world of hepatobiliary surgery from the clinical and scientific standpoint. Until most updated or higher levels of evidence are disclosed, we feel appropriate to recommend that surgeons and institutions willing to develop or expand a LLR program should follow the guidance of such fruitful expert consensus with careful consideration of their local resources and personal experiences.

After the first reports in the early ' 90 s, LLR on anterolateral segments have been rapidly up taken. The reasonable compromise between technical complexity and clinical efficacy has acted as an engine for pioneer surgeons to gain experience and become expert centers, with the subsequent diffusion of these procedures even in non-tertiary referral units. However, as remarked by Cai, the actual scenery of laparoscopic resections includes procedures of great difficulty as hemihepatectomies, caudate lobe resections, anatomical segmentectomies, resections on the posterosuperior segments up to conventional two stage hepatectomies and ALPPS. Various technical adjustments have been described to accomplish these challenging resections. The well-known caudal approach for major resections, first described by Gayet, represents the most acknowledged conceptual change for this technique. Few years ago, we have also described a systematic choice of approach to laparoscopic right hemihepatectomy based on the liver texture, size and position of the lesion and closeness to the hepatocaval confluence (5). For deeply and centrally located liver lesions, the "diamond technique" has also 
been described to decrease the difficulty of parenchymasparing resections associated with laparoscopy (6). The abovementioned technical expedients exemplify that the laparoscopic approach to liver resections imposes the surgeon to a set of theoretical knowledge and practical skills different from open surgery, which needs to be systematized and standardized as much as possible to facilitate an orderly learning curve towards increasingly difficult procedures.

Hepatocellular carcinoma and colorectal liver metastases remain the predominant indications. Especially for cirrhotic patients, the advantages of minimally invasiveness in terms of reduced ascites and liver decompensation may also apply to patients with baseline advanced liver impairment. In this setting, it has been demonstrated that the postoperative course of selected Child B patients receiving a LLR may be not different to Child $\mathrm{A}$, and that portal hypertension is not a risk factor for postoperative major complications when a liver resection in cirrhosis is performed by laparoscopy (7). Also, the benefits for other oncological indications are increasingly being reported (8). Recently evidence on the advantages of LLR for patients with metastases from breast cancer has been published, highlighting a faster return to adjuvant treatments (9). The advantages have to be interpreted also in the setting of different procedures. Despite a traditional definition of major hepatectomies, a recent large multicenter study has pointed out the different benefits and outcomes for laparoscopic right and left hemihepatectomies when separately compared with their respective open (10). With an even more focused analysis, we have recently published evidence on a significant differential benefit between laparoscopic anterolateral and posterosuperior resections compared with their open counterparts: while both resulting beneficial when compared to open, the advantages of laparoscopy appear greater for posterosuperior than anterolateral resections despite their technical complexity (11). The degree of advantage provided by laparoscopy has to be studied more in detail for different type of resections, keeping in mind that the most difficult seems to be associated with the greatest clinical benefits.

As far as concerns the operative strategies, the use of methods for inflow control remains a crucial point to ensure the feasibility and efficacy of liver resections, especially when performed by laparoscopy. The limitation of blood loss keeps the surgical field clean and dry, thus helping to conduct safe and oncologically efficient resections even in those locations most hostile to laparoscopic instruments. Several studies have reported the safety of an intermittent
Pringle maneuver even in diseased parenchyma. In addition, a wide use of an intermittent clamping (either systematic or on demand) has been described by a large number of centers with extensive experience and regular activity with LLR without any alert on perceived detrimental effects of associating the Pringle maneuver to the pneumoperitoneal pressure. We are in full agreement with the latest recommendations which regard the Pringle maneuver as the most effective method for the inflow control, and stress the possibility to take advantage of a selective hemihepatic control when convenient.

Regarding technical aspects, we totally agree on the central and non-replaceable role of intraoperative ultrasound, which is well defined in LLR as well as in open especially for cancer patients $(2,12)$. We also recall that advanced vision technologies, real-time indocyanine green fluorescence imaging and augmented reality navigation systems are additional elements applicable to LLR. Their role should be considered helpful to accomplish especially the most difficult resections, as they may compensate several technical challenges as the lack of palpation, favor the recognition of structures, facilitate correct transection planes and detect sources of biliary spillage. Instead, the area of ideal application of robotics in liver surgery remains still to be determined. Since their perioperative efficacy appears similar to that of pure laparoscopy and the robotic approach retains increased costs, further studies are advocated to optimize their adoption and allocation (3).

The issue of cost-effectiveness of LLR deserves a specific focus, since their impact on financials may be significant. It should be considered that several studies have already demonstrated the economic advantages associated with laparoscopic minor resections, mainly related to the beneficial postoperative course. The evidence of costneutrality for major or complex liver resections needs to be fine-tuned. Notwithstanding a recent study has provided an estimate of not negligible costs associated with conversion, the adoption of a pure laparoscopic approach for major laparoscopic liver resection still appears without cost disadvantage compared to open (13). In addition, more evidence is needed to determine the financial impact of LLR in low income countries.

We conclude congratulating with Dr. Cai for the nice update on the status of LLR in China. The efforts put by Chinese surgeons and centers into laparoscopic liver surgery are well known to the worldwide community of hepatobiliary surgery: commendable examples of procedures have been presented at international meetings, and relevant publications 
are part of the core literature on the topic. We take this opportunity to mirror the development in the far East with the continuous progress and spreading of LLR in Italy (14). As for China, our commitment is witnessed by various publications from tertiary referral centers and, more recently, by studies on many topics arising from the Italian prospective registry on laparoscopic liver resections (I GO MILS) (15).

\section{Acknowledgments}

Funding: None.

\section{Footnote}

Conflicts of Interest: All authors have completed the ICMJE uniform disclosure form (available at http://dx.doi. org/10.21037/hbsn.2019.11.07). The authors have no conflicts of interest to declare.

Etbical Statement: The authors are accountable for all aspects of the work in ensuring that questions related to the accuracy or integrity of any part of the work are appropriately investigated and resolved.

Open Access Statement: This is an Open Access article distributed in accordance with the Creative Commons Attribution-NonCommercial-NoDerivs 4.0 International License (CC BY-NC-ND 4.0), which permits the noncommercial replication and distribution of the article with the strict proviso that no changes or edits are made and the original work is properly cited (including links to both the formal publication through the relevant DOI and the license). See: https://creativecommons.org/licenses/by-nc-nd/4.0/.

\section{References}

1. Cai X. Laparoscopic liver resection: the current status and the future. Hepatobiliary Surg Nutr 2018;7:98-104.

2. Abu Hilal M, Aldrighetti L, Dagher I, et al. The Southampton Consensus Guidelines for Laparoscopic Liver Surgery: From Indication to Implementation. Ann Surg 2018;268:11-8.

3. Liu R, Wakabayashi G, Kim HJ, et al. International consensus statement on robotic hepatectomy surgery in 2018. World J Gastroenterol 2019;25:1432-44.

4. Han HS, Cho JY, Kaneko H, et al. Expert Panel Statement on Laparoscopic Living Donor Hepatectomy. Dig Surg 2018;35:284-8.

5. Ratti F, Cipriani F, Catena M, et al. Approach to hepatocaval confluence during laparoscopic right hepatectomy: three variations on a theme. Surg Endosc 2017;31:949.

6. Cipriani F, Shelat VG, Rawashdeh M, et al. Laparoscopic Parenchymal-Sparing Resections for Nonperipheral Liver Lesions, the Diamond Technique: Technical Aspects, Clinical Outcomes, and Oncologic Efficiency. J Am Coll Surg 2015;221:265-72.

7. Cipriani F, Fantini C, Ratti F, et al. Laparoscopic liver resections for hepatocellular carcinoma. Can we extend the indication in cirrhotic patients? Surg Endosc 2018;32:617-26.

8. Martinez-Cecilia D, Cipriani F, Vishal S, et al. Laparoscopic versus open liver resection for colorectal metastases in elderly and octogenarian patients: a multicenter propensity score based analysis of short- and long-term outcomes. Ann Surg 2017;265;1192-200.

9. Ratti F, Serenari M, Zanello M, et al. Appraisal of diseasespecific benefits of minimally invasiveness in surgery of breast cancer liver metastases. J Surg Oncol 2019;120:1169-76.

10. Cipriani F, Alzoubi M, Fuks D, et al. Pure laparoscopic versus open hemihepatectomy: a critical assessment and realistic expectations. A propensity score-based analysis of right and left hemihepatectomies from 9 European tertiary referral centers. J Hepatobiliary Pancreat Sci 2019. [Epub ahead of print].

11. Cipriani F, Ratti F, Paganelli M, et al. Laparoscopic or open approaches for posterosuperior and anterolateral liver resections? A Propensity Score based analysis of the degree of advantage. HPB 2019;21:1676-86.

12. Ferrero A, Lo Tesoriere R, Russolillo N. Ultrasound liver map technique for laparoscopic liver resections. World J Surg 2019;43:2607-11.

13. Cipriani F, Ratti F, Cardella A, et al. Laparoscopic Versus Open Major Hepatectomy: Analysis of Clinical Outcomes and Cost Effectiveness in a High-Volume Center. J Gastrointest Surg 2019;23:2163-73.

14. Aldrighetti L, Ratti F, Cillo U, et al. Diffusion, outcomes and implementation of minimally invasive liver surgery: a snapshot from the I GoMILS (Italian Group of Minimally Invasive Liver Surgery) Registry. Updates Surg 2017;69:271-83.

15. Ardito F, Aldrighetti L, Guglielmi A, et al. Surgical Management of Hepatic Benign Disease: Have the Number of Liver Resections Increased in the Era of Minimally Invasive Approach? Analysis from the I Go MILS (Italian Group of Minimally Invasive Liver Surgery) Registry. J Gastrointest Surg 2019. [Epub ahead of print].

Cite this article as: Cipriani F, Ratti F, Aldrighetti L. Laparoscopic liver resections at the gates of 2020: a standalone field of hepatobiliary surgery. HepatoBiliary Surg Nutr 2020;9(3):371-373. doi: 10.21037/hbsn.2019.11.07 